\title{
EFFECTIVE RECONSTRUCTION OF GENERIC GENUS 4 CURVES FROM THEIR THETA HYPERPLANES
}

\author{
DAVID LEHAVI
}

\begin{abstract}
Effective reconstruction formulas of a curve from its theta hyperplanes are known classically in genus 2 (where the theta hyperplanes are Weierstrass points), and 3 (where, for a generic curve, the theta hyperplanes are bitangents to a plane quartic). However, for higher genera, no formula or algorithm are known. In this paper we give an explicit (and simple) algorithm for computing a generic genus 4 curve from it's theta hyperplanes.
\end{abstract}

\section{INTRODUCTION}

The quest for methods of reconstructing a curve from its theta hyperplanes goes back to the 19th and early 20th century geometers Aronhold and Coble: in the non hyperelliptic genus 3 case, theta hyperplanes are simply bitangents, and both Aronhold and Coble provided formulas for reconstructing curves from certain ordered subsets of the 28 bitangents of the curve (see $[\mathrm{A}],[\mathrm{Co}]$ chapter IV, and [Dol] sections 6.1.2 and 6.2.2).

Recent years witnessed some revived interest in generalizations of this problem from several directions: first relaxing the need for ordered theta hyperplanes (see [CS1], [L]), and then generalizations to higher genus curves (see [CS2]) and abelian varieties (see [GS-M1], [GS-M2]). However for $g>3$, these results are not effective. In this work we give an effective result for the generic genus 4 case. Our result assumes ordered theta characteristic; that being said, by [CS2] this requirement is redundant for generic curves.

Throughout this paper we consider a generic complex curve $C$ of genus 4 ; since $C$ is generic we assume that all its odd theta characteristics are 1 dimensional - i.e. if $\theta$ is an odd theta characteristic of $C$ then $\operatorname{dim} H^{0}(\theta, C)=$ 1. Hence, for each odd theta characteristic $\theta$ there exists a unique hyperplane $l_{\theta}$ - called a theta hyperplane - in the dual canonical system of $C$ such that when $C$ is identified with it's canonical image, the points in the intersection product $C \cdot l_{\theta}$ are all double, and the points in $\frac{1}{2} C \cdot l_{\theta}$ sum up to $\theta$. This hyperplane is the projectivization of the plane $T_{\theta} \Theta_{C} \subset T_{\theta} J C$ under the identification of $T_{\theta} J C=T_{0} J C=H^{0}\left(K_{C}\right)^{*}$. Recall (see e.g. [Dol] 5.4.2), that if $\alpha$ is a non-trivial 2 torsion point on the Jacobian $J C$, then the Steiner

Date: January 11, 2021.

1991 Mathematics Subject Classification. 14H40, 14Q20. 
system $\Sigma_{C, \alpha}$ of the pair $(C, \alpha)$ is defined to be the set

$$
\left\{\theta: 2 \theta=K_{C} \text { and } \operatorname{dim} H^{0}(\theta, C)=\operatorname{dim} H^{0}(\theta+\alpha, C) \equiv 1 \quad \bmod 2\right\} .
$$

The number of theta characteristics in a Steiner system of a genus $g$ curve is $2^{g-1} \cdot\left(2^{g-1}-1\right)$; i.e. in our case a Steiner system is comprised of $8 \cdot 7=56$ odd theta characteristics, out of the total of $2^{4-1}\left(2^{4}-1\right)=120$ odd theta characteristics of the Jacobian. For each pair $\theta, \theta+\alpha$, and corresponding theta hyperplanes $l_{\theta}, l_{\theta+\alpha}$, we let $q_{\{\theta, \theta+\alpha\}} \in\left|\mathcal{O}_{\left|K_{C}\right|^{*}}(2)\right|$ be the image of $\left\{l_{\theta}, l_{\theta+\alpha}\right\} \in S^{2}\left|K_{C}\right|$ under the embedding: $S^{2}\left|K_{C}\right| \rightarrow\left|\mathcal{O}_{\left|K_{C}\right|^{*}}(2)\right|$.

We set $V_{C}:=H^{0}\left(\mathcal{O}_{\left|K_{C}\right|^{*}}(2)\right)$, and denote the map $H^{0}\left(\mathcal{O}_{\left|K_{C}+\alpha\right|^{*}}(2)\right) \rightarrow$ $H^{0}\left(2 K_{C}\right)$ by $i$, the projection $V_{C} \rightarrow H^{0}\left(2 K_{C}\right)$ by $p$, and the pre-image $p^{-1} i H^{0}\left(\mathcal{O}_{\left|K_{C}+\alpha\right|^{*}}(2)\right) \subset V_{C}$ by $V_{C, \alpha}$. Finally, we denote the projectivization of a linear space by $\mathbb{P}$. The first component of our reconstruction algorithm is given by the following:

Theorem 1. Let $C, \alpha, V_{C}$, and $V_{C, \alpha}$ be as above, (specifically, recall that $C$ is generic), and let $Q_{C}$ be the unique quadric surface containing the canonical image of $C$, then

(1) for all $\alpha \in J C[2] \backslash\{0\}$ we have

$$
\operatorname{span}\left(\left\{q_{\{\theta, \theta+\alpha\}}\right\}_{\theta \in \Sigma_{C, \alpha}}\right)=\mathbb{P} V_{C, \alpha} ;
$$

(2) moreover, $\cap_{\alpha \in J C[2] \backslash\{0\}} \mathbb{P} V_{C, \alpha}=\left\{\left[Q_{C}\right]\right\}$, where $\left[Q_{C}\right]$ denotes the moduli point representing $Q_{C}$ in the space $\mathbb{P} V_{C}$.

Note that the theorem may be rephrased in the following "genus free" terms: the intersection $\cap_{\alpha \in J C[2] \backslash\{0\}} \operatorname{span}\left(\left\{q_{\{\theta, \theta+\alpha\}}\right\}_{\theta \in \Sigma_{C, \alpha}}\right)$ is the locus of quadrics over $\left|K_{C}\right|^{*}$ containing $C$. The beauty in this statement arises from its relation to the Enriques-Babbage Theorem (see e.g. [ACGH] chapter VI $\S 3)$, which states that a canonical curve is either trigonal, or is isomorphic to a plane quintic, or is cut out by quadrics.

The proof of the theorem eventually reduces to the analysis of bi-elliptic curves: the first part is proved by considering a (degenerated) double cover of the nodal cubic; whereas the second part is proved by considering a curve with a big automorphism group.

Turning our attention back to the genus 4 case, we now aim to locate an irreducible cubic surface containing the canonical image of the curve $C$. To this end we will make heavy use of the following classical Theorem:

Theorem (Wirtinger - see $[\mathrm{W}]$, [Co] chapter $\mathrm{V}$, or $[\mathrm{Ca}]$ ). Let $C$ be a generic genus 4 complex curve, and let $\alpha$ be a non trivial 2-torsion point on the Jacobian of $C$. Then the image of $C$ in the Prym canonical system $\left|K_{C}+\alpha\right|^{*}$ is a sextic with 6 nodes, which are the intersection points of four lines in general position: $l_{1}, l_{2}, l_{3}, l_{4}$ (not to be confused with the hyperplanes $l_{\theta}$; we will never use the two notations in the same context). Moreover, let $S$ be the blow-up of $\left|K_{C}+\alpha\right|^{*}$ at the six intersection points $l_{i} \cap l_{j}$, let $H$ be the pullback to $S$ of a generic hyperplane in $\left|K_{C}+\alpha\right|^{*}$ and let $E$ be the exceptional 
divisor in $S$, then the complete linear system $\left|\mathcal{O}_{S}(3 H-E)\right|$ is canonically isomorphic to $\left|K_{C}\right|$. Finally, the image of $S$ in the dual of this linear system - which we will denote below by $W_{C, \alpha}$ - is a Cayley cubic (the unique space cubic with four nodes), where the four nodes are the blow downs of the strict transforms in $S$ of the four lines $l_{1}, \ldots, l_{4}$, and where by its definition $W_{C, \alpha}$ contains the canonical image of $C$.

Armed with this theorem we can state the following:

Theorem 2. The four hyperplanes through each of the four triplets of nodes of $W_{C, \alpha}$ are - set theoretically - the four intersection points in $\mathbb{P} V_{C}$ of the six dimensional projective subspace

$$
\mathbb{P}\left(\left(V_{C}^{*} /\left(\left(V_{C} / V_{C, \alpha}\right)^{*} \wedge\left(V_{C} / V_{C, \alpha}\right)^{*}\right)\right)^{*}\right),
$$

and the 2nd Veronese image of $\left|K_{C}\right|$ in $\mathbb{P} V_{C}$. Moreover, each of these four points has multiplicity 2 in the intersection.

Given Theorems 1 and 2, the canonical image of the curve $C$ is readily reconstructed as the intersection of $Q_{C}$ and $W_{C, \alpha}$ - which is the only cubic with nodes at the four intersection points of triplets of the four hyperplanes determined in Theorem 2.

\section{Acknowledgment.}

Sam Grushevsky and an anonymous reviewer read early versions of this work and gave the author extremely valuable feedback.

\section{Proof of THEOREM 1}

We start by analyzing special Steiner systems of bi-elliptic curves. Below we denote the ramification (resp. branch) locus of a map - by $R_{-}$(resp. $\left.B_{-}\right)$.

Theorem 3 (Coble, [Co] chapter IV). Let $\pi: C_{b e} \rightarrow E$ be a bi-elliptic double cover where the genus of $C_{b e}$ is 4 . We first note that $C_{b e}$ is not hyperelliptic. Let $\pi$ be the involution induced by $\pi$ on the dual canonical system $\left|K_{C_{b e}}\right|^{*}$ (which is a projective linear involution). Then the fixed spaces of $\bar{\pi}$ are a hyperplane $H_{\pi}$, and a point which we call the focal point. Identifying $C_{b e}$ with its canonical image, the intersection $H_{\pi} \cdot C_{b e}$ is the ramification divisor $R_{\pi}$, which is comprised of six distinct points; the image of $C$ in $H_{\pi}$ under the projection from the focal point is $E$ embedded as a plane cubic; $H_{\pi}$ is naturally identified with a $g_{3}^{2}$ on $E$, denoted by $|L|^{*}$ which satisfied $2 L \sim B_{\pi}$.

Conversely, the curve $C_{b e}$ can be reconstructed from such data of $E, B_{\pi}$ (comprised of six distinct points), and $L$ in the following way: Construct $\mathbb{P}^{3}$ as a cone over $|L|^{*}$, and in it reconstructs $C_{b e}$ as the intersection of two surfaces:

- the cone $\tilde{E}$ over the image of $E$ in the linear system $|L|^{*}$, and,

- a quadric surface $\tilde{Q}$ in $\mathbb{P}^{3}$ ramified over $|L|^{*}$ at the unique conic satisfying intersecting $E$ at $B_{\pi}$. 
Proof. We first prove, arguing by contradiction, that $C_{b e}$ is not hyperelliptic. Suppose it is, and denote the hyperelliptic system on it by $\left|H_{C_{b e}}\right|$. Since the hyperelliptic involution commutes with all other involutions, we would have two double covers $f:\left|H_{C_{b e}}\right|^{*} \rightarrow \mathbb{P}^{1}$ and $g: E \rightarrow \mathbb{P}^{1}$ such that $C_{b e}=$ $\left|H_{C_{b e}}\right|^{*} \times_{\mathbb{P}^{1}} E$. However, we then have the following inequality in DivE:

$$
B_{\pi} \leq f^{*} B_{g}
$$

which is impossible since $\operatorname{deg} B_{g}=2 \Rightarrow \operatorname{deg} f^{*} B_{g}=4$, while $\operatorname{deg} B_{\pi}=6$.

Note that since $\pi$ is a double cover, $B_{\pi}$ is a sum of six distinct points. We proceed to analyze the action of $\bar{\pi}$ : As $K_{E}$ is trivial, the image of $R_{\pi}$ in $\left|K_{C_{b e}}\right|^{*}$ is cut out by some hyperplane $H_{\pi}$. Moreover, since $C_{b e}$ does not admit a $g_{2}^{1}$, at most 3 of the 6 points of $R_{\pi}$ are collinear. Since 6 points on $H_{\pi}$, no 4 of which are collinear, are fixed by $\bar{\pi}$, the entire plane $H_{\pi}$ is also fixed by $\bar{\pi}$.

Recall that a projective linear involution which admits a fixed hyperplane also admit a fixed point out of this hyperplane. We call this point the focal point and project the dual canonical system $\left|K_{C_{b e}}\right|^{*}$ from this point to $H_{\pi}$. As $\bar{\pi}$ is linear, all the lines through the focal point (and some point on $H_{\pi}$ ) are $\bar{\pi}$ invariant. Hence, the intersections of the canonical image of $C_{b e}$ with these lines are exactly the fibers of $\pi$, and the degree 2 map $C_{b e} \rightarrow H_{\pi}$ factors through a map $E \rightarrow H_{\pi}$; moreover, as $C_{b e}$ is a degree 6 embedding, the induced map $E \rightarrow H_{\pi}$ is a degree 3 embedding. Setting $L \in \operatorname{Div}^{3} E$ to be the (pullback to $E$ under an the embedding of the) intersection of the image of $E$ in $H_{\pi}$ and some line in $H_{\pi}$, we may identify $H_{\pi}$ with the dual complete linear system $|L|^{*}$. Moreover, since the images of the $B_{\pi}$ in $|L|^{*}$ is the intersection of the image of $E$ in $H_{\pi}$, and the quadric surface $Q_{C_{b e}}$, they sit on a conic in $H_{\pi}$; thus, we have $2 L \sim B_{\pi}$ in $\operatorname{Pic}^{6} E$. Expressing $\left|K_{C_{b e}}\right|^{*}$ as a cone over $|L|^{*}$, we see that $C_{b e}$ is the intersection of the cone over the image of $E$ in $|L|^{*}$ through the focal point, and the quadric surface ramified over $|L|^{*}$ at the unique conic passing through the images of $B_{\pi}$ there.

Henceforth, we will identify $E$ with it's image in $|L|^{*}$, and $C_{b e}$ with it's canonical image. We now turn to the identification of some of the theta hyperplanes of $C_{b e}$ :

Proposition-Definition 4. Assuming $C_{b e}$ does not admit a theta null, there are exactly 24 theta hyperplanes of $C_{b e}$ invariant under the bi-elliptic involution. They are given as follows: Denote the 6 distinct points of $B_{\pi}$ by $b_{1}, \ldots, b_{6}$; for each $b_{i}$ let $x_{i 1}, x_{i 2}, x_{i 3}, x_{i 4}$ be the four points in $E$ satisfying $2 x_{i j}+b_{i} \sim L$. For each $i, j$ we denote by $l_{i j}$ the line which satisfies $l_{i j} \cdot E=$ $2 x_{i j}+b_{i}$. Let $H_{i j}$ be the pullback of $l_{i j}$ to $\left|K_{C_{b e}}\right|^{*}$ under the identification of $\left|K_{C_{b e}}\right|^{*}$ as a cone over $|L|^{*}$, then

$$
H_{i j} \cdot C_{b e}=\pi^{-1}\left(2 x_{i j}+b_{i}\right) .
$$

This proposition is an explicit form of proposition 2 in [B], where the genera of the curves involved are 4 and 1 . 
Proof. Identifying $C_{b e}$ with it's canonical image we have $H_{i j} \cdot C_{b e}=2 \pi^{-1} x_{i j}+$ $\pi^{-1} b_{i}$; hence $H_{i j}$ represents an effective theta characteristic. Since we assume that $C_{b e}$ does not admit a theta null, $H_{i j}$ is a theta hyperplane.

Conversely, assume that $H$ is a theta hyperplane invariant under the bielliptic involution, and let $l$ be the projection of $H$ to the linear system $|L|^{*}$, then the following properties hold:

- If some $b_{i}$ satisfies $E \cdot l>b_{i}$, then $H \cdot C_{b e}>\pi^{-1}\left(b_{i}\right)$, which is the ramification point lying over $b_{i}$ - with multiplicity 2 .

- If some point $y \neq b_{1} \ldots, b_{6}$ satisfies $l \cdot E>a y$ for some positive $a$, then both points in $\pi^{-1}(y)$ are in the intersection product $H \cdot C_{b e}$, each with intersection multiplicity $a$.

Thus, if the intersection product $l \cdot E$ contains $B$ branching points and $n$ other points with positive intersection multiplicities $a_{k}$, then

$$
2\left(B+a_{1}+\cdots+a_{n}\right)=\# C_{b e} \cdot H=6, \quad \text { where all the } a_{i} \text { s are even. }
$$

The case where $n=0$ is the case where $Q_{C_{b e}} \cap H_{\pi}$ contains the line $l$, which implies that this intersection is the union of two lines, which implies that $Q_{C_{b e}}$ is singular, which implies that $C_{b e}$ has a theta null. Whence, we have only one possible solution: $B=n=1, \quad a_{1}=2$.

Proposition 5. Let $\beta$ be a non trivial 2 torsion point in JE, then for each $i=1, \ldots, 6$ and $j=1,2,3,4$ there is some $j^{\prime}$ such that $x_{i j}-x_{i j^{\prime}}=\beta$. This is a pairing on the $x_{i j} s$, which induces a pairing on the $H_{i j} s$. Moreover, denoting by $\pi^{*}$ the map $\operatorname{Pic}(E) \rightarrow \operatorname{Pic}\left(C_{b e}\right)$ induced by $\pi$, we have $\pi^{*} x_{i j}-$ $\pi^{*} x_{i j^{\prime}}=\pi^{*} \beta \in J C_{b e}[2] \backslash\{0\}$.

Proof. For each $i, j$ the shifts of $x_{i, j}$ by the four points of $J E[2]$ give the four $x_{i, j^{\dagger}}$, where $j^{\dagger}=1,2,3,4$. Hence, $\beta$ induces a natural partition to pairs $x_{i j}, x_{i j^{\prime}}$, so that $x_{i j}-x_{i j^{\prime}}=\beta$ for all $i, j$. The second part follows since $\pi^{*}$ is an embedding.

In the proof of Corollary 8 below, as well as in the proof of Theorem 1 we will apply a restricted form the following:

Proposition 6 (Degeneracy loci of maps between vector bundles). The degeneracy loci of a map between two vector bundles over a base scheme are closed subschemes of the base.

This proposition, as well as the proof, are classical. The proposition follows from a choice of a local basis to the bundles - which is possible since the statement of the proposition is local, from induction on the degeneracy rank, and from the fact that the determinant is trivial on a closed sub scheme.

As we just indicated, we will not apply the full strength of Proposition 6, but rather the following corollary: 
Corollary 7. Let $\mathcal{V} / X$ be a vector bundle over a base $X$, and let $\mathcal{V}_{1}, \ldots \mathcal{V}_{n}$ be sub-bundles of $\mathcal{V}$. Then the function $\operatorname{dim}\left\langle\left.\mathcal{V}_{1}\right|_{x}, \ldots,\left.\mathcal{V}_{n}\right|_{x}\right\rangle$ is lower semicontinuous on $X$, and the function $\operatorname{dim}\left(\left.\cap_{i=1}^{n} \mathcal{V}_{i}\right|_{x}\right)$ is upper semi-continuous on $X$.

Proof. By induction it suffices to prove this claim for two sub-bundles. By Proposition 6 the degeneracy locus of the map $\mathcal{V}_{1} \oplus \mathcal{V}_{2} \rightarrow \mathcal{V}$ is a closed subscheme of the base. Since the degeneracy loci of both $\mathcal{V}_{1} \rightarrow \mathcal{V}$ and $\mathcal{V}_{2} \rightarrow \mathcal{V}$ are empty by definition (as they are sub-bundles), we are done.

Lemma 8. Let $E, L, \beta, b_{i}, \ldots b_{6}$, and $x_{i j}$ be as above and generic, then the twelve reducible conics $l_{i j} \cup l_{i j^{\prime}}$ in $|L|^{*}$ span the 5 dimensional space $\left|\mathcal{O}_{|L|^{*}}(2)\right|$.

Note that Lemma 8 has nothing to do with $C_{b e}$; it is a statement about plane cubics per se.

Proof. We apply the lower semi-continuity of the dimension of span from Corollary 7 . We consider the degenerated case where $E$ is a nodal cubic, and $\beta$ is degenerated to the trivial 2 -torsion point. As a model for the nodal cubic we will use the plane cubic $E_{0}$ given by the nulls of $x^{3}+y^{3}-x y z$. The isomorphism between $\mathbb{C}^{*}$ and $E_{0}$ is given by

$$
\phi: t \mapsto\left(-t: t^{2}: 1-t^{3}\right) .
$$

The generic "combinatorial" scenario, where we have 24 points $x_{i j}$ coming in quartets indexed by the $j$ coordinates, where the differences $x_{i j_{1}}-x_{i j_{2}}$ are the four 2 -torsion points and where $x_{i j}-x_{i j^{\prime}}=\beta$, degenerates in the $E_{0}$ case to the following scenario: We have 12 "doubled" points $x_{i j}$ which come in pairs indexed by the $j$ coordinate, where $x_{i 1}, x_{i 2}=\phi\left( \pm t_{i}\right)$ for some $t_{i}$, and where each $x_{i j}$ is paired with itself. As in the generic case the six points $b_{i}$ have to sit on a conic; however, as for this degenerated case we have $b_{i}=\phi\left(t_{i}^{2}\right)$, this constrain now translates to the easier constrain: $\prod_{i=1}^{6} t_{i}^{2}=1$. Finally, instead of $24 l_{i j}$ s we now have 12, where each one is "trivially paired" with itself. These $12 l_{i j}$ s are given by

$$
\begin{aligned}
T_{E_{0}}(\phi(t)) & =\left.\left(3 x^{2}-y z: 3 y^{2}-x z:-x y\right)\right|_{x=-t, y=t^{2}, z=1-t^{3}} \\
& =\left(3 t^{2}-t^{2}\left(1-t^{3}\right): 3 t^{4}+t\left(1-t^{3}\right):-t^{3}\right) \sim\left(2 t+t^{4}: 1+2 t^{3}:-t^{2}\right),
\end{aligned}
$$

for $t= \pm t_{1}, \ldots, \pm t_{6}$. We will show that if $t_{1}^{2}, \ldots, t_{5}^{2}$ are all distinct, then the span of the "doubled" $T_{E_{0}}\left( \pm t_{i}\right)$ for $i=1, \ldots, 5$ is the entire space $\left|\mathcal{O}_{\left|\mathbb{P}^{2}\right|^{*}}(2)\right|$.

Squaring the projective linear form $T_{E_{0}}(t)$ we get (using the lexicographic order on degree 2 monomials):

$$
\left(4 t^{2}+4 t^{5}+t^{8}: 2 t+5 t^{4}+2 t^{7}:-2 t^{3}-t^{6}: 1+4 t^{3}+4 t^{6}:-t^{2}-2 t^{5}: t^{4}\right) .
$$

Let

$$
\begin{aligned}
v_{t} & :=\left(4 t^{2}+4 t^{5}+t^{8}, 2 t+5 t^{4}+2 t^{7},-2 t^{3}-t^{6}, 1+4 t^{3}+4 t^{6},-t^{2}-2 t^{5}, t^{4}\right) \\
& =\left(4 t^{2}+t^{8}, 5 t^{4},-t^{6}, 1+t^{6},-t^{2}, t^{4}\right)+t\left(4 t^{4}: 2,-2 t^{2}+2 t^{6}, 4 t^{2},-2 t^{5}, 0\right),
\end{aligned}
$$


then our aim is to show that the evaluations of $v_{t}$ at $\pm t_{1}, \pm t_{5}$ span the 6 dimensional affine space $H^{0}\left(\mathcal{O}_{|L|^{*}}(2)\right)$. Taking $t \neq 0, \infty$, the linear span of $v_{t_{i}}, v_{-t_{i}}$ is equal to the linear span of $\frac{1}{2}\left(v_{t_{i}}+v_{-t_{i}}\right), \frac{1}{2}\left(v_{t_{i}}-v_{-t_{i}}\right)$. Denoting $s=t^{2}$, the vectors $\frac{1}{2}\left(v_{t_{i}}+v_{-t_{i}}\right), \frac{1}{2}\left(v_{t_{i}}-v_{-t_{i}}\right)$ are given by

$$
\left(4 s+s^{4}, 5 s^{2},-s^{3}, 1+s^{3},-s, s^{2}\right), \quad t\left(2 s^{2}, 1,-s+s^{3}, 2 s,-s^{2}, 0\right)
$$

respectively. We now consider the matrix whose rows are the evaluations of the vector $\left(4 s+s^{4}, 5 s^{2},-s^{3}, 1+s^{3},-s, s^{2}\right)$ at five distinct values of $s$. Performing the column operations

$$
\mathrm{Col}_{1} \mapsto \mathrm{col}_{1}+4 \mathrm{col}_{5}, \quad \mathrm{Col}_{4} \mapsto \mathrm{Col}_{4}+\mathrm{col}_{3}, \quad \mathrm{col}_{2} \mapsto \mathrm{col}_{2}-5 \mathrm{col}_{6}
$$

on this matrix we get the matrix whose rows are the evaluations of the vector $\left(s^{4}, 0,-s^{3}, 1,-s, s^{2}\right)$. Except for the second (trivial) column, and up to permutations and sign changes of the columns, this matrix is a Vandermonde matrix; thus it is of degree 5 for any 5 distinct values of $s$. Since the rows of the last matrix span the kernel of the operator $v \mapsto v(0,1,0,0,0,0)^{t}$, retracing the column operations we performed on the original matrix, we see that the span of the rows is the kernel of the operator $v \mapsto v(0,1,0,0,0,-5)^{t}$.

Finally, as

$$
t\left(2 s^{2}, 1,-s+s^{3}, 2 s,-s^{2}, 0\right)(0,1,0,0,0,-5)^{t}=t \neq 0 \quad \text { generically, }
$$

we see that for five distinct non zero values of $t^{2}$, the projective space spanned by the squares of the 10 of the $l_{i j}$ s is 5 dimensional.

Corollary 9. Let $C_{b e}, E, b_{i}, L, \beta, x_{i j}$ be generic as above. Then the map

$$
\begin{aligned}
\left|\mathcal{O}_{|L|^{*}}(2)\right| & \rightarrow \mathbb{P} V_{C_{b e}} \\
{[q] } & \mapsto[\text { the cone over } q \text { through the focal point }]
\end{aligned}
$$

is an embedding into $\mathbb{P} V_{C_{b e}, \pi^{*} \beta}$, whose image is spanned by the twelve $q_{\left\{\theta, \theta+\pi^{*} \beta\right\}}$ 's corresponding to the partition, described in Proposition-Definition 4, of the 24 hyperplanes $H_{i j}$ to twelve pairs.

Proof. The map is an embedding since $\left|K_{C_{b e}}\right|^{*}$ is a cone over $|L|^{*}$. By Lemma $8,\left|\mathcal{O}_{|L|^{*}}(2)\right|$ is spanned by the twelve reducible conics $l_{i j} \cup l_{i j^{\prime}}$; hence by Proposition-Definition 4, the image is spanned by the twelve $q_{\left\{\theta, \theta+\pi^{*} \beta\right\}} \mathrm{S}$ described above. Finally, the twelve $q_{\left\{\theta, \theta+\pi^{*} \beta\right\}}$ 's lie in $\mathbb{P} V_{C_{b e}, \pi^{*} \beta}$ by their definition; hence, so does their span.

Remark 10 (An alternative view of Corollary 9). As $Q_{C_{b e}}$ contains the curve $C_{b e}$, projecting $\mathbb{P} V_{C_{b e}, \pi^{*} \beta}$ from $\left[Q_{C_{b e}}\right]$ we get $\mathbb{P} V_{C_{b e}, \pi^{*} \beta}$. Composing this projection on the embedding from Corollary 9, we get an isomorphism between $\left|\mathcal{O}_{|L|^{*}}(2)\right|$ and $\mathbb{P} V_{C_{b e}, \pi^{*} \beta}$. In fact, more is true: in [LR] (Theorem 2.9 in the journal version, or 2.15 in the arxiv version) it is proved, by a careful analysis of Coble's construction, that there is a natural isomorphism between $\left|\mathcal{O}_{|L+\beta|^{*}}\right|$ and $\left|\mathcal{O}_{\left|K_{C_{b e}}+\pi^{*} \beta\right|^{*}}\right|$. 
Proof of part 1 of Theorem 1. In Corollary 9 we showed that for $C_{b e}, E, \beta$ as in the corollary there are exactly twelve quadrics $q_{\theta, \theta+\pi^{*} \beta}$ passing through the focal point, and that these quadrics span a five dimensional subspace of $\mathbb{P} V_{C_{b e}, \pi^{*} \beta}$. As the Steiner system $\Sigma_{C_{b e}, \pi^{*} \beta}$ admits $56-24=32$ theta hyperplanes which do not pass through the focal point of the involution, there are 16 quadrics $q_{\theta, \theta+\pi^{*} \beta}$ which do not sit on the 5 dimensional space spanned by the 12 quadrics from 4 . Hence, $\operatorname{dim} \operatorname{span}\left(\left\{q_{\left\{\theta, \theta+\pi^{*} \beta\right\}}\right\}_{\theta \in \Sigma_{C_{b e}, \pi^{*} \beta}}\right) \geq 6$. By Corollary 7 we see that $\operatorname{dim} \operatorname{span}\left(\left\{q_{\{\theta, \theta+\alpha\}}\right\}_{\theta \in \Sigma_{C, \alpha}}\right) \geq 6$ for generic curves, and not only for bi-elliptic ones. However, as $\operatorname{dim} \operatorname{span}\left(\left\{q_{\{\theta, \theta+\alpha\}}\right\}_{\theta \in \Sigma_{C, \alpha}}\right) \subset$ $\mathbb{P} V_{C, \alpha}$, which is 6 dimensional, we see that for a generic curve $C, \operatorname{dim} \mathbb{P} V_{C, \alpha}=$ 6.

To prove the second part of theorem 1 we analyze the mutual structure of several Steiner systems on one curve. As we have already analyzed (some of) the structure of a specific Steiner system on a bi-elliptic curve, we will introduce and analyze below a special genus 4 curve with many bi-elliptic involutions; this would immediately give us many Steiner systems on this curve, of the form already analyzed above.

Proposition-Definition 11 (Kuribayahi and Kuribayashi, see [KK] Proposition $2.4(\mathrm{f})(1))$. There is exactly one genus 4 curve, denoted here by $C_{9 \times 8}$, whose automorphism group is isomorphic to $(\mathbb{Z} / 3)^{2} \ltimes D_{8}$. Endowing the dual canonical system of $C_{9 \times 8}$ with the coordinates $x_{1}, \ldots x_{4}$, and presenting the automorphism group as automorphism of the canonical system, the group is generated by the automorphisms:

$$
\begin{aligned}
& \tau_{1}=\left(x_{1}: x_{2}: x_{3}: x_{4}\right) \mapsto\left(\omega x_{1}: \omega^{2} x_{2}: x_{3}: x_{4}\right), \quad \text { where } \omega^{3}=1, \\
& \tau_{2}=\left(x_{1}: x_{2}: x_{3}: x_{4}\right) \mapsto\left(-x_{2}:-x_{1}: x_{3}: x_{4}\right), \quad \text { and } \\
& \tau_{3}=\left(x_{1}: x_{2}: x_{3}: x_{4}\right) \mapsto\left(x_{3}: x_{4}: x_{1}: x_{2}\right) .
\end{aligned}
$$

Proof. See $[\mathrm{KK}]$. Verifying that the automorphism group contains a group isomorphic to $(\mathbb{Z} / 3)^{2} \ltimes D_{8}$, which is all we need for our purpose here, is immediate using the explicitly model in Corollary 12 below.

Corollary 12 (Swinarski, see [S]). The canonical image of the curve $C_{9 \times 8}$, in the coordinates of 11, is the intersection of the following cubic and quadric:

$$
x_{1}^{3}-x_{2}^{3}+x_{3}^{3}-x_{4}^{3}, \quad x_{1} x_{2}+x_{3} x_{4} .
$$

Proof. Direct verification.

Proposition 13. The quotient of $C_{9 \times 8}$ under $\tau_{2}$ is elliptic. Denoting the quotient by $\tau_{2}$ by $\pi_{\tau_{2}}$ and using our notations from Theorem 3, the focal point is $(1: 1: 0: 0)$ and $H_{\pi_{\tau_{2}}}=\{(a:-a: b: c) \mid a, b, c \in \mathbb{C}\}$. The image of the elliptic quotient by $\tau_{2}$ in $H_{\pi_{\tau_{2}}}$ is given by the null set of $(2 a)^{3}+b^{3}-$ $c^{3}-3(2 a) b c$.

Proof. The claim about the invariant sub-spaces follows immediately by direct verification. By Corollary $12, C_{9 \times 8}$ sits on the null set of the cubic 
surface

$x_{1}^{3}-x_{2}^{3}+x_{3}^{3}-x_{4}^{3}-3\left(x_{1}-x_{2}\right)\left(x_{1} x_{2}+x_{3} x_{4}\right)=\left(x_{1}-x_{2}\right)^{3}+x_{3}^{3}-x_{4}^{3}-3\left(x_{1}-x_{2}\right) x_{3} x_{4}$.

The plane cubic curve is the intersection of this cone and the invariant hyperplane; i.e. in the plane coordinates given above it is the curve

$$
(2 a)^{3}+b^{3}-c^{3}-3(2 a) b c .
$$

Proof of part 2 of Theorem 1. By definition, for any $C, \alpha$ we have

$$
\left[Q_{C}\right] \in \cap_{\alpha \in J C \backslash\{0\}} \mathbb{P} V_{C, \alpha} .
$$

As $V_{C, \alpha}$ are sub-bundles of $V_{C}$ over the moduli of $C, \alpha$, Corollary 7 tells us that in order to proof part 2 of Theorem 1 we need only prove it for the curve $C_{9 \times 8}$.

If $\sigma$ is an involution of $C_{9 \times 8}$, we will denote by $\alpha_{\sigma}$ one of the three 2torsion points in $J C_{9 \times 8}[2] \backslash\{0\}$ invariant under the involution: these are the pullback of the three non trivial 2-torsion points of the quotient by $\sigma$, which is an elliptic curve ("morally", we don't care which one of the three we pick is that as we are dealing with second symmetric products of linear systems). By Corollary 9, (and using the notation $L$ as in the corollary), the space $V_{C_{9 \times 8}, \alpha_{\tau_{2}}}$ is spanned by the pullback of $S^{2} H^{0}(L)$ to $S^{2} H^{0}\left(K_{C_{9 \times 8}}\right)=V_{C_{9 \times 8}}$ under the presentation of $\left|K_{C_{9 \times 8}}\right|^{*}$ as a cone over $|L|^{*}$, and a quadric form in $V_{C_{9 \times 8}, \alpha_{\tau_{2}}}$ which is not trivial on the focal point; as the quadric $Q_{C_{9 \times 8}}$ does not pass through the focal point, and is in $V_{C_{9 \times 8}, \alpha_{\tau_{2}}}$, we may choose this quadric form to be $x_{1} x_{2}+x_{3} x_{4}$.

In the notations used in $11, L$ is spanned by $x_{1}-x_{2}, x_{3}, x_{4}$; hence:

$$
\begin{aligned}
V_{C_{9 \times 8}, \alpha_{\tau_{2}}} & =\left\langle x_{1} x_{2}+x_{3} x_{4},\left(x_{1}-x_{2}\right)^{2},\left(x_{1}-x_{2}\right) x_{3},\left(x_{1}-x_{2}\right) x_{4}, x_{3}^{2}, x_{3} x_{4}, x_{4}^{2}\right\rangle \\
& =\left\langle x_{1}^{2}+x_{2}^{2},\left(x_{1}-x_{2}\right) x_{3},\left(x_{1}-x_{2}\right) x_{4}, x_{1} x_{2}, x_{3}^{2}, x_{3} x_{4}, x_{4}^{2}\right\rangle .
\end{aligned}
$$

By symmetry,

$$
V_{C_{9 \times 8}, \alpha_{\tau_{2} \tau_{1}}}=\left\langle x_{1}^{2}+\omega^{2} x_{2}^{2},\left(x_{1}-\omega x_{2}\right) x_{3},\left(x_{1}-\omega x_{2}\right) x_{4}, x_{1} x_{2}, x_{3}^{2}, x_{3} x_{4}, x_{4}^{2}\right\rangle .
$$

In order to compute the intersection of these spaces, we observe that $V_{C 9 \times 8}$ may be broken to the direct sum of the following spaces:

$$
\left\langle x_{1}^{2}, x_{2}^{2}\right\rangle \oplus\left\langle x_{1} x_{3}, x_{2} x_{3}\right\rangle \oplus\left\langle x_{1} x_{4}, x_{2} x_{4}\right\rangle \oplus\left\langle x_{1} x_{2}, x_{3}^{3}, x_{3} x_{4}, x_{4}^{2}\right\rangle .
$$

Considering the spanning elements (indeed - bases) we chose for the spaces $V_{C_{9 \times 8}, \alpha_{\tau_{2}}}$ and $V_{C_{9 \times 8}, \alpha_{\tau_{2} \tau_{1}}}$, it is clear that both spaces are direct sums of their intersections with the components in equation $(\diamond)$, that both intersections with the last component in equation $(\diamond)$ form the entire last component, and finally that the intersections of these spaces with each of the other three components in equation $(\diamond)$ are one dimensional and different from each other. Hence we have

$$
V_{C_{9 \times 8}, \alpha_{\tau_{2}}} \cap V_{C_{9 \times 8}, \alpha_{\tau_{2} \tau_{1}}}=\left\langle x_{1} x_{2}, x_{3}^{2}, x_{3} x_{4}, x_{4}^{2}\right\rangle=\left\langle x_{1} x_{2}+x_{3} x_{4}, x_{3}^{2}, x_{3} x_{4}, x_{4}^{2}\right\rangle .
$$


To conclude the proof, note that the intersection of this space with it's image under $\tau_{3}$ is generated just by $x_{1} x_{2}+x_{3} x_{4}$, and recall that the space generated by $x_{1} x_{2}+x_{3} x_{4}$ is by definition in the intersection of all the $V_{C_{9 \times 8}, \alpha}$ 's.

\section{Proof of THEOREM 2}

Lemma 14. Let $X$ be the intersection of the image of $\left|K_{C}\right| \times\left|K_{C}\right|$ in $\mathbb{P} V_{C}$ with $\mathbb{P} V_{C, \alpha}$, then the two dimensional fibers of the projection on the first coordinate of the pullback of $X$ to $\left|K_{C}\right| \times\left|K_{C}\right|$ lie exactly over the moduli points which represent the four hyperplanes through the four possible triplets of the blow-downs of the strict transforms in $S$ of the four lines $l_{1}, \ldots, l_{4}$ (see Wirtinger's Theorem in the introduction regarding $S$ and the $l_{i} s$ ).

Proof. Note that the isomorphism $\left|\mathcal{O}_{S}(3 H-E)\right| \cong\left|K_{C}\right|$ from Wirtinger's theorem induces an isomorphism between quadric forms over $\left|K_{C}\right|^{*}$, and sextic forms over $\left|K_{C}+\alpha\right|^{*}$ containing the six intersection points $l_{i} \cap l_{j}$. Moreover, if we let $L_{i}$ be a linear form on $\left|K_{C}+\alpha\right|^{*}$ whose null set is $l_{i}$, then this map sends any quadric form on $\left|K_{C}\right|^{*}$ which is zero on the four nodes of $W_{C, \alpha}$ to a product of the four $L_{i}$ 's and a quadric in $\left|K_{C}+\alpha\right|^{*}$.

We write the cubic $W_{C, \alpha}$ as the null set:

$$
W_{C, \alpha}=Z\left(h_{1} h_{2} h_{3}+h_{1} h_{2} h_{4}+h_{1} h_{3} h_{4}+h_{2} h_{3} h_{4}\right),
$$

where $h_{1}, h_{2}, h_{3}, h_{4}$ are linear forms such that the four points $\cap_{1 \leq i \leq 4, i \neq j} Z\left(h_{i}\right)$ - for $j \in\{1,2,3,4\}$ - are the four nodes of $W_{C, \alpha}$. We endow the space $V_{C}$ with the coordinates $\left\{h_{i} h_{j}\right\}_{1 \leq i \leq j \leq 4}$. Then the quadrics passing through the four nodes of $W_{\alpha}$ are exactly the null sets of quadric forms in the span of the six forms: $\left\{h_{i} h_{j}\right\}_{1 \leq i<j \leq 4}$.

Recall that the curve $C$ is generic on the moduli of genus 4 curves, which is 9 dimensional. Hence, writing $Q_{C}$ as the null-set of $q_{C}=\sum_{1 \leq i<j \leq 4} d_{i j} h_{i} h_{j}+$ $\sum_{i=1}^{4} a_{i} h_{i}^{2}$ (not to be confused with the $q_{\theta, \theta+\alpha}$ which already appeared before), $q_{C}$ has ten free parameters; thus w.l.o.g. we may assume that all the $a_{i}$ s are non 0 . As $q_{C}$ is not in the span of $\left\{h_{i} h_{j}\right\}_{1 \leq i<j \leq 4}$, the space $V_{C, \alpha}$ is spanned inside $V_{C}$ by $q_{C}$ and $\left\{h_{i} h_{j}\right\}_{1 \leq i<j \leq 4}$. We can now compute the intersection $\mathbb{P} V_{C, \alpha}$ with the image of the map:

$$
s:\left|K_{C}\right| \times\left|K_{C}\right| \rightarrow \mathbb{P} V_{C} .
$$

Let $\sum_{i=1}^{4} b_{i} h_{i}$ and $\sum_{i=1}^{4} c_{i} h_{i}$ be two canonical sections, then their product modulo $\operatorname{span}\left(\left\{h_{i} h_{j}\right\}_{1 \leq i<j \leq 4}\right)$ is $\sum_{i=1}^{4} b_{i} c_{i} h_{i}^{2}$. Hence, in order for the projectivization of the product of these canonical sections to lie in $\mathbb{P} V_{C, \alpha}$ we must have an equality

$$
\left(b_{1} c_{1}, b_{2} c_{3}, b_{3} c_{3}\right)=k\left(a_{1}, a_{2}, a_{3}, a_{4}\right) \text { for some } k .
$$

If all the $b_{i}$ s are non 0 , then the only non trivial solution to this equality is the one where $k \neq 0$, and $c_{i}=k a_{i} / b_{i}$. If at least one $b_{i}$ is 0 then $k$ is necessarily also 0 , and in this case the indices for which the $b_{i}$ s are 0 are the one complimentary (in the set $\{1, \ldots, 4\}$ ) to the ones for which the $c_{i}$ s are 0 . 
Pulling the image of the intersection back to $\left|K_{C}\right| \times\left|K_{C}\right|$ (under the map $s$ ), we see that the projection on the first coordinate is a birational isomorphism between the intersection and $\left|K_{C}\right|$, where the exceptional fibers are of two types:

- The fibers over each of the moduli points $\left[h_{i}\right]$ is a $\mathbb{P}^{2}$.

- The fiber over each point in the six lines $\operatorname{span}\left(\left[h_{i}\right],\left[h_{j}\right]\right)$, is a $\mathbb{P}^{1}$.

Proof of theorem 2. By Lemma 14 the four hyperplanes through triplets of the four nodes of $W_{C, \alpha}$ are exactly the projective points represented by $a \in$ $H^{0}\left(K_{C}\right)$, such that for all non trivial $v \in\left(V_{C} / V_{C, \alpha}\right)^{*} \subset V_{C}^{*}=S^{2}\left(H^{0}\left(K_{C}\right)\right)$, the null spaces of the operators

$$
\begin{aligned}
H^{0}\left(K_{C}\right) & \rightarrow \mathbb{C} \\
x & \mapsto v \cdot(a \otimes x), \quad \text { where } \cdot \text { is tensor contraction }
\end{aligned}
$$

are identical. Equivalently, this means that any two vectors in $\left(V_{C} / V_{C, \alpha}\right)^{*} \cdot a$ are dependant I.e. the solutions are the solutions of the equation:

$$
\left(\left(V_{C} / V_{C, \alpha}\right)^{*} \cdot a\right) \wedge\left(\left(V_{C} / V_{C, \alpha}\right)^{*} \cdot a\right)=0,
$$

However, since contraction commutes with tensor products (and wedge products, which may be viewed as tensor products followed by projections to a subspace),

$$
\left(\left(V_{C} / V_{C, \alpha}\right)^{*} \cdot a\right) \wedge\left(\left(V_{C} / V_{C, \alpha}\right)^{*} \cdot a\right)=\left(\left(V_{C} / V_{C, \alpha}\right)^{*} \wedge\left(V_{C} / V_{C, \alpha}\right)^{*}\right) \cdot(a \otimes a) .
$$

Hence the solution set is the intersection of

$$
\mathbb{P}\left(\left(V_{C}^{*} /\left(\left(V_{C} / V_{C, \alpha}\right)^{*} \wedge\left(V_{C} / V_{C, \alpha}\right)^{*}\right)\right)^{*}\right),
$$

and the image of $\left|K_{C}\right|^{*}$ under the 2nd Veronese map. Finally, Since the degree of the 2 nd Veronese of a 3 dimensional space is $2^{3}=8$, and since by Theorem $\mathrm{C}$ in [Ca], the Galois group acting on the four nodes of $W_{C, \alpha}$ is $S_{4}$; hence, each of the intersection points has multiplicity 2 .

\section{REFERENCES}

[A] Aronhold, S. Über den gegenseitigen Zusamemmenhang der 28 Doppeltangenten einer allgemeiner Curve 4ten Grades, Monatberichter der Akademie der Wissenschaften zu Berlin, 1864, 499-523.

[ACGH] Arbarello, E.; Cornalba, M.; Griffiths, P. A.; Harris, J. Geometry of algebraic curves. Vol. I. Grundlehren der Mathematischen Wisse nschaften, 267. SpringerVerlag, 1985.

[B] Beauville, A. Vanishing thetanulls on curves with involutions. Rendiconti del Circolo Matematico di Palermo 2013, V. 62, Issue 1, 61-66.

[CS1] Caporaso, L; Sernesi, E. Recovering plane curves from their bitangents. J. Algebraic Geom. 12 (2003), no. 2, 225-244.

[CS2] Caporaso, L.; Sernesi, E. Characterizing curves by their odd theta-characteristics. J. Reine Angew. Math. 562 (2003), 101-135.

[Ca] Catanese, F. On the rationality of certain moduli spaces related to curves of genus 4. Algebraic geometry (Ann Arbor, Mich., 1981), 30-50, Lecture Notes in Math., 1008 
[Co] Coble, A. Algebraic geometry and Theta functions. Revised printing. Amer. Math. Soc. Coll. Pub., vol. X Amer. Math. Soc., 1961.

[Dol] Dolgachev, I. Classical algebraic geometry: a modern view. Cambridge Univ. Press. (2012).

[GS-M1] Grushevsky, S.; Salvati-Mani, R On the gradient of odd theta functions., J. reine. angew. Math 573 (2004), 45-59.

[GS-M2] Grushevsky, S.; Salvati-Mani, R Theta functions of arbitrary order and their derivatives., J. reine, angew. Math 590 (2006), 31-43.

[KK] Kuribayashi, A.; Kuribayashi, I. Automorphism groups of compact Riemann surfaces of genera three and four J. Pure Appl. Algebra 65 (1990), no. 3, 63-79

[L] Lehavi, D. Any smooth plane quartic can be reconstructed from its bitangents. Israel Journal of Mathematics Volume 146, Number 1 (2005) 371-379.

[LR] Lehavi, D.; Ritzenthaler, C. An explicit formula for the arithmetic-geometric mean in genus 3. Experiment. Math. 16 (2007), no. 4, 421-440.

$[\mathrm{R}]$ Recillas, S. Jacobians of curves with $g_{4}^{1} s$ are the Pryms of trigonal curves. Bol. Soc. Mat. Mexicana (2) 19 (1974), 9-13.

[S] Swinarski, D. Equations of curves with automorphisms, available online at http://www.math.uga.edu/ davids/sf.pdf

[W] Wirtinger, W. Untersuchungen über Abel'sche Functionen vom Geschlechte 3. Math. Ann. 40 (1892), no. 2, 261-312.

E-mail address: dlehavi@gmail.com 